\title{
Analysis and Application of Repetitive Control Scheme for Three-Phase Active Power Filter with Frequency Adaptive Capability
}

\author{
Biaoguang Sun ${ }^{\dagger}$, Yunxiang Xie*, Hui Ma* and Li Cheng*
}

\begin{abstract}
Active power filter (APF) has been proved as a flexible solution for compensating the harmonic distortion caused by nonlinear loads in power distribution power systems. Digital repetitive control can achieve zero steady-state error tracking of any periodic signal while the sampling points within one repetitive cycle must be a known integer. However, the compensation performance of the APF would be degradation when the grid frequency varies. In this paper, an improved repetitive control scheme with frequency adaptive capability is presented to track any periodic signal with variable grid frequency, where the variable delay items caused by time-varying grid frequency are approximated with Pade approximants. Additionally, the stability criterion of proposed repetitive control scheme is given. A three-phase shunt APF experimental platform with proposed repetitive control scheme is built in our laboratory. Simulation and experimental results demonstrate the effectiveness of the proposed repetitive control scheme.
\end{abstract}

Keywords: Active power filter, Repetitive control, Variable grid frequency, Pade approximants

\section{Introduction}

Power quality has become a serious concern in power distribution power system due to harmonic currents pollution caused by various nonlinear loads such as adjustable speed drives, arc furnaces and other power electronic-related devices. These harmonic currents increase the power losses, deteriorate the quality of the voltage waveform, and may cause resonance problem. Active power filter (APF) that operates as controllable current sources and provides fast dynamic response to load variations has been widely used to suppress the harmonic current produced by nonlinear loads. APF has been proved to be a flexible solution to suppress the harmonic currents due to its simplicity and effectiveness [1,2].

The key technology of the APF system is tracking of the periodic reference signals. To solve this issue, numerous current control strategies have been introduced, such as hysteresis control [3], proportional-integral (PI) control [4], proportional-resonant (PR) control [5-6], repetitive control [7-8], etc. PI control has good tracking capability for DC and low-frequency signals. However, the reference signals are composed of many frequency components (fundamental and its multiples) in APF. The PI control is not an optimum solution for APF, due to the limitation of control bandwidth and the adverse effect of delay time. Repetitive control technique, based on internal model principle (IMP) [9], is particularly suitable for this situation since it introduces infinite gains at the interested harmonic frequencies. The

\footnotetext{
$\dagger$ Corresponding Author: School of Electrical Power, South China University of Tech, China. (sunbiaoguang@163.com)

* School of Electrical Power, South China University of Tech, China. (yxxie@scut.edu.cn, \{446724497,49452332\}@qq.com)

Received: August 6, 2015; Accepted: November 23, 2015
}

composite control strategy combing repetitive control with PI feedback control is an effective approach for APF. PI controller is utilized to enhance the dynamic response to the load variations. Repetitive controller is adopted to improve the steady-state accuracy. The faster dynamic performance of PI controller will not be affected by the slower repetitive controller. Nowadays, this control structure has been widely adopted in power electronicrelated equipments, such as uninterruptible power supply (UPS) systems [10], unity power factor rectifiers [11], Pulse-Width Modulation (PWM) inverters [12] and distributed generation [13].

In repetitive control, the signal generator $z^{-N} / 1-z^{-N}$ must be included [7]. $N=f_{s} / f$ is the order of the repetitive controller, $f_{s}$ is the sampling rate, $f$ is the fundamental frequency of the reference signal. Repetitive control can achieve zero steady-state error tracking of any periodic reference signals when $N$ is a known integer [14]. However, the grid frequency is time-varying within a certain range, for instance $49.5 \sim 50.5 \mathrm{~Hz}$. The order of repetitive control often contains a decimal at a fixed sampling rate [14-15]. Therefore, the compensation performance of APF would have a great recession when conventional repetitive control technology is employed.

Ensuring the $N$ keeps a known integer in the presence of grid frequency variations, several approaches have been introduced to solve this problem. These solutions could be divided into two groups: fixed sampling rates and variable sampling rates. Variable sampling rates [16-17] can keep the constant sampling points within one repetitive control cycle by adjusting the changed sampling period with the grid frequency variations. However, the discrete control plant model would change with the sampling period varies. 
In the literatures [18-19], authors introduce a special designed finite impulse response (FIR) filter, which is cascaded with a traditional delay time item, to replace the conventional low-pass filter (LPF) with function for system stabilization. In the literatures $[14,20]$, the fractional-order filter is approximated by a Lagrange Interpolation method with an integer number. Their coefficients can be easily updated online. However, this method need higher order approximants to maintain repetitive control open-loop gains without attenuation. In the literature [21], authors used Pade approximants to approximate the fractional delay item in repetitive control.

This paper presents an improved repetitive control scheme with frequency adaptive capability to enhance the steady-state compensation accuracy. The variable-delay items in repetitive controller are approximated by using Pade approximants. Their coefficients can be earlier updated online and the nominal part keeps a constant. So, the proposed control scheme can track any periodic signal with variable grid frequency. A shunt APF experimental system is built in our laboratory. Various simulation and experimental results demonstrate the feasibility and effectiveness of the proposed repetitive control scheme.

\section{Repetitive Control Scheme with Frequency Adaptive Capability}

\subsection{Conventional repetitive control scheme}

A typical closed-loop current control system with a plugin repetitive control [18] is illustrated in Fig. 1, where $G_{r}(s)$ is the internal model, $r(s)$ is the reference input, $u_{r}(s)$ is the output of repetitive control module, $y(s)$ is the output, $e(s)=r(s)-y(s)$ is the tracking error, $d(s)$ is the disturbance, $G_{p}(s)$ is the control plant model, and $G_{c}(s)$ is the PI feedback controller with high-enough robustness margin. $Q(s)$ can be designed to a constant or a LPF to enhance the stability margin of the control system. $G_{f}(s)$ being the user defined compensator to ensure closed-loop stability.

According to Fig. 1, the transfer function of internal model can be described as

$$
G_{r}(s)=\frac{e^{-T_{r} s}}{1-e^{-T_{r} s}}
$$

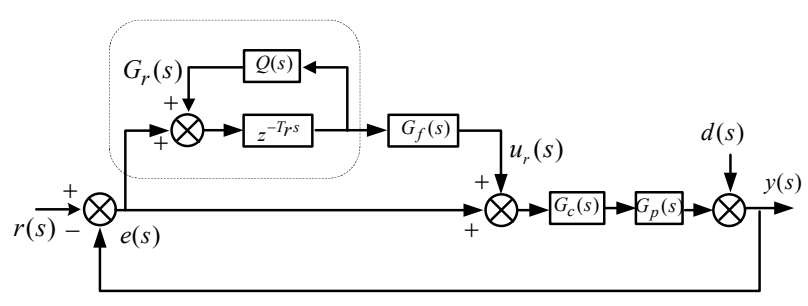

Fig. 1. Plug-in repetitive control system. where $T_{r}=N T_{S}$ being the time delay period. $N$ being the sampling points within one repetitive cycle. $T_{s}$ being the sampling period.

From Fig. 1, the tracking error $e(s)$ without repetitive control can be described as

$$
e_{0}(s)=\frac{r(z)-d(s)}{1+G_{c}(s) G_{p}(s)}
$$

and tracking error with repetitive control becomes

$$
e(s)=e_{0}(s) \frac{1-Q(s) e^{-T_{r} s}}{1-\left[Q(s)-G_{f}(s) P(s)\right] e^{-T_{r} s}}
$$

where $P(s)=\left(G_{c}(s) G_{p}(s)\right) /\left(1+G_{c}(s) G_{p}(s)\right)$ is the equivalent control plant of repetitive controller.

The sufficient stability criterion of Fig. 1 is given as follows [7-8]:

1) The closed-loop system without repetitive controller $P(s)$ is stable.

2) $\|Q(s)\|_{\infty}<1$.

3) $\left\|Q(s)-G_{f}(s) P(s)\right\|_{\infty}<1$.

The internal model, $G_{r}(s)$, can be expanded as [22]

$$
G_{r}(s)=-\frac{1}{2}+\frac{1}{T_{r} s}+\frac{1}{T_{r}} \sum_{h=1}^{\infty} \frac{s}{s^{2}+(h \varpi)^{2}}
$$

where $\varpi=2 \pi f$ being the fundamental angular frequency, $h$ being the order of harmonic frequencies. As previous earlier, the repetitive control has a good tracking performance if the $N$ is a known integer. (4) indicates that the repetitive controller is able to compensate all harmonic distortion under nominal grid frequency, as shown in Fig. 2(a).

However, the grid frequency $f$ is always time-varying within a certain range in practical system. Therefore, the order $N$ would contain a fraction at a fixed sampling rate. The resonance points of the conventional repetitive control will deviate from the actual fundamental frequency and interested harmonic frequencies. Then, the performance of the control system will be degenerated. Fig. 2(b) shows the Bode plot of the conventional repetitive controller with different fundamental frequency, $L_{1}(49.5 \mathrm{~Hz}), L_{2}(50 \mathrm{~Hz})$ and $L_{3}(50.5 \mathrm{~Hz})$. Fig. 2 indicates that, if the fundamental frequency deviated from $50 \mathrm{~Hz}$, conventional repetitive controller cannot exactly compensate harmonic currents with $(50 \pm 0.5) h \mathrm{~Hz}(h=1,2, \ldots)$, since they are low gains at these frequencies. In other words, conventional repetitive control scheme is sensitively for variable grid frequency. Meanwhile, Fig. 2(b) indicates that the repetitive controller would be able to compensate the time-varying fundamental 


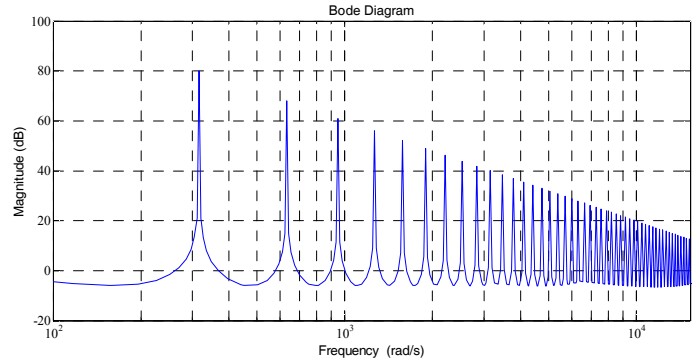

(a)

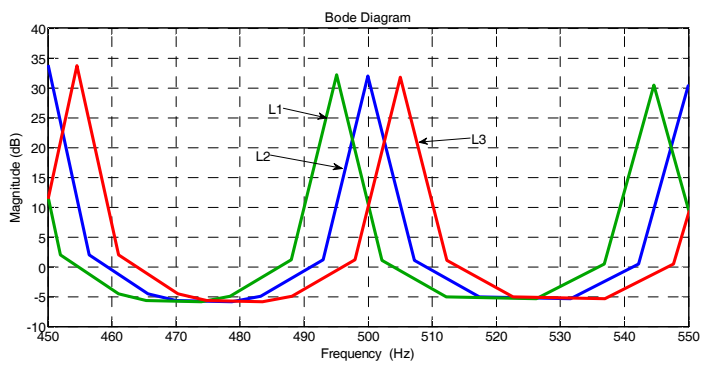

(b)

Fig. 2. Bode plot of the conventional repetitive controller: (a) With nominal grid frequency; (b) With different grid frequency.

and its harmonic frequencies if the resonance points move to the appropriate position following with the variable grid frequency.

\subsection{Proposed repetitive control scheme with frequency- adaptive capability}

Assuming that $Q(s)$ is constant, the block diagram of the internal model principle is shown in Fig. 3. When the grid frequency $f$ varies, which is a common phenomenon in practical power grid, the $N$ cannot maintain an integer, namely, the time delay period $T_{r}$ will change.

Defining $D=192$ as the nominal part of $N$, and $d$ as its offset part, i.e.

$$
\begin{gathered}
T_{r}=N T_{s}=(D+d) T_{s} \\
G_{r}(s)=\frac{e^{-s N T_{S}}}{1-e^{-s N T_{S}}}=\frac{e^{-s D T_{S}} e^{-s d T_{S}}}{1-e^{-s D T_{S}} e^{-s d T_{S}}}
\end{gathered}
$$

Where $d \in\left[d_{\text {min }}, d_{\text {max }}\right]$ is bounded, since the grid frequency is time-varying within a certain range.

The exponential function $e^{-s d T_{S}}$ can be closely approximated by Pade approximants [23] as follows:

$$
e^{-s d T_{S}}=\frac{b_{0}+b_{1} s d T_{s}+\cdots+b_{m}\left(s d T_{s}\right)^{m}}{a_{0}+a_{1} s d T_{s}+\cdots+a_{m}\left(s d T_{s}\right)^{m}}
$$

where $a_{j}=\frac{(2 m-j) !}{(2 m) !}, \quad b_{j}=(-1)^{j} \frac{(2 m-j) !}{(2 m) !}, j \in[0, m]$.

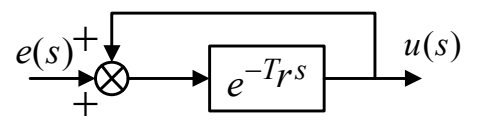

(a)

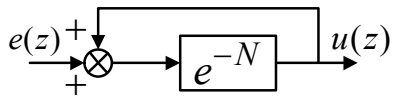

(b)

Fig. 3. Block diagram of the internal model principle: (a) Time domain; (b) Discrete-time domain.
With increasing in the degree $m$, a more accurate approximation can be acquired. When $m=1$, (6) can be written as

$$
e^{-s d T_{S}}=\frac{1-\frac{1}{2} s d T_{S}}{1+\frac{1}{2} s d T_{S}}
$$

which can be discretized using Tustin discretization method as

$$
s=\frac{2}{T_{S}} \frac{z-1}{z+1}
$$

Substituting (8) into (7), the (7) can be rewritten as

$$
e^{-s d T_{S}}\left(z, \frac{2}{T_{S}} \frac{z-1}{z+1}\right)=d(z)=\frac{(1-d)+(1+d) z^{-1}}{(1+d)+(1-d) z^{-1}}
$$

So (5) in discrete-time can be rewritten as

$$
G_{r}(z)=\frac{z^{-D} d(z)}{1-z^{-D} d(z)}
$$

The proposed repetitive control scheme with frequency adaptive capability is illustrated in Fig. 4, where the internal model is replaced by $(10) . G_{f}(z)$ can be designed as $k_{r} z^{k} S(z), k_{r}$ is the repetitive control gain, $z^{k}$ is the phase lead term, $S(z)$ is a user defined compensator to correct magnitude characteristic of the plant.

Fig. 5 shows the Bode plot of open-loop repetitive controller with fixed sampling period under different grid frequency, $L_{1}(50 \mathrm{~Hz}, d=0), L_{2}(49.5 \mathrm{~Hz}, d=1.939)$ and $L_{3}(50.5 \mathrm{~Hz}, d=-1.901)$. The magnitude-frequency responses show that the internal model $G_{r}(z)$ can provide enough gain to suppress the actual harmonic components with variable fundamental frequency. The open-loop gains do not attenuate, which just offset the resonant points with the variation of grid frequency. 


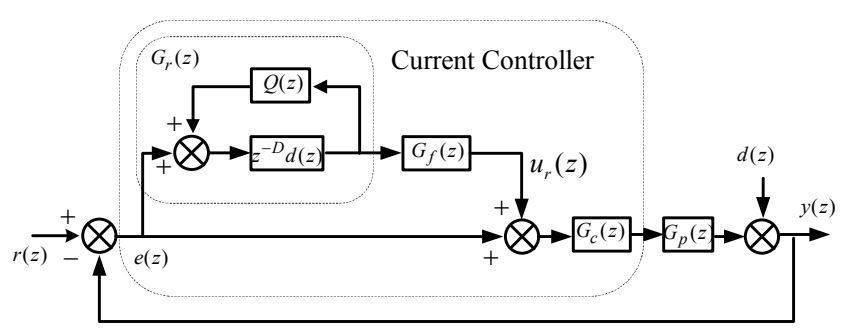

Fig. 4. Block diagram of the proposed repetitive control with frequency-adaptive capability.

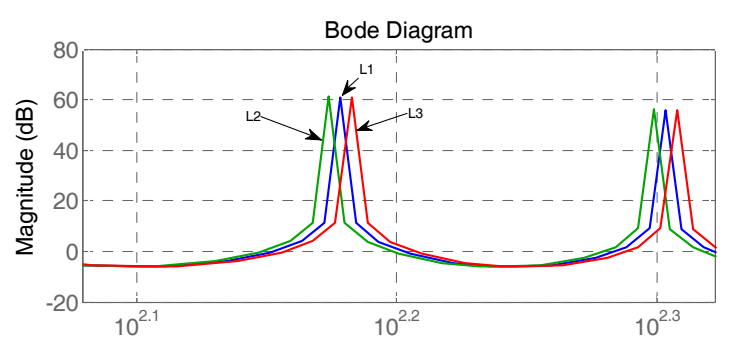

(a)

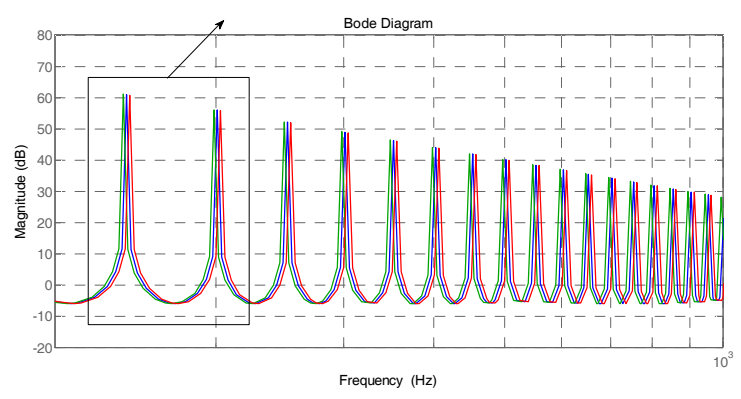

(b)

Fig. 5. Bode plot of proposed repetitive controller.

From Fig. 4, the tracking error $e(z)$ of repetitive control can be described as

$$
\frac{e(z)}{r(z)}=\frac{1-Q(z) z^{-D} d(z)}{1-\left[Q(z)-k_{r} z^{k} S(z) P(z)\right] z^{-D} d(z)}
$$

The characteristic equation of the proposed repetitive control system is

$$
1-\left[Q(z)-k_{r} z^{k} S(z) P(z)\right] z^{-D} d(z)=0
$$

The necessary and sufficient condition for system stability is that $N$ roots of (12) are inside the unity circle. According to the small gain theorem [24], a sufficient condition for system stability can be described as

$$
|H(z)|=\left|\left(Q(z)-k_{r} z^{k} S(z) P(z)\right)\right|\left|z^{-D} d(z)\right|<1
$$

in which $z=e^{j \omega T_{S}}$, and $\omega \in\left[0, \pi / T_{S}\right] . T_{S}$ is the sampling time and $\pi / T_{S}$ is the Nyquist frequency. The $d(z)$ can be regarded as constants. $\left|z^{-D} d(z)\right| \rightarrow 1$. (13) means that: when $\omega$ increases from zero to the Nyquist frequency, if the end of the vector $k_{r} e^{j \omega k T_{S}} S\left(e^{j \omega k T_{S}}\right) P\left(e^{j \omega k T_{S}}\right)$ does not exceed the unity circle at the end of $Q\left(e^{j \omega k T_{S}}\right)$, the repetitive control system is sufficiently stable. So the stability criterion of (13) would be almost the same as that of the conventional repetitive control. The stability range of the proposed control gain is $0<k_{r}<2$, which is the same as that of the conventional repetitive control.

\section{Three-Phase Shunt APF with Frequency Adaptive Capability}

\subsection{Problem description}

A complete system circuit structure of three-phase shunt APF with LCL output filter is illustrated in Fig. 6(a). The shunt APF operates as a controllable current source parallel with nonlinear diode rectifier loads connected at the point common coupling (PCC). The subscript $k$ denotes phase $a, b$ and $c . u_{s k}$ is the grid voltage, $u_{k}$ is the voltage at the PCC, the system inductance $L_{s}$ is normally neglected due to its low value relatively, thus $u_{s k}=u_{k} . i_{s k}, i_{l k}$ and $i_{f k}$ are the grid, load and converter current of each phase. $L_{f f}$ is the converter-side filter inductors, $L_{g f}$ is the grid-side filter inductors, $C_{f}$ is the filter capacitors, and $R_{d}$ is the damping resistors. $C$ and $V_{d c}$ are the dc-link capacitor and voltage, respectively.

Fig. 6(b) shows the block diagram of the proposed control scheme with frequency adaptive capability for three-phase shunt APF. The control system contains a phase-locked loop (PLL), a dc-link voltage regulator with PI control, a reference current calculator and the proposed current controller. The PLL is used to produce the phase information $\theta$ of the grid voltage and the ratio $N$ of the sampling frequency to the grid frequency. The dc-link voltage regulator forces the dc-link voltage $V_{d c}$ to track the reference voltage $V_{d c r e f}$. The reference current calculator detects the harmonic components of grid currents and produces reference current signals. The proposed current controller with grid voltage feed-forward and output current feedback cross decoupling is shown in Fig. 6 (b).

The implementation of proposed repetitive controller in DSP is shown in Fig. 6 (c), where $e(c u r)$ is the current error, $e(c u r-1)$ is the error of last control period, $e_{1}(c u r)$ is the error of last time delay period, $u_{r 1}(c u r)$ is the repetitive error of last time delay period, $u_{r}(\mathrm{cur})$ is the output of repetitive controller. The register array is updated after calculation of current control period. 


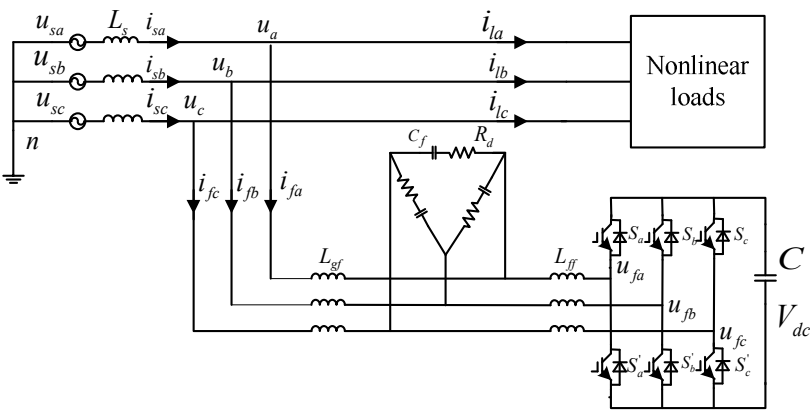

(a)

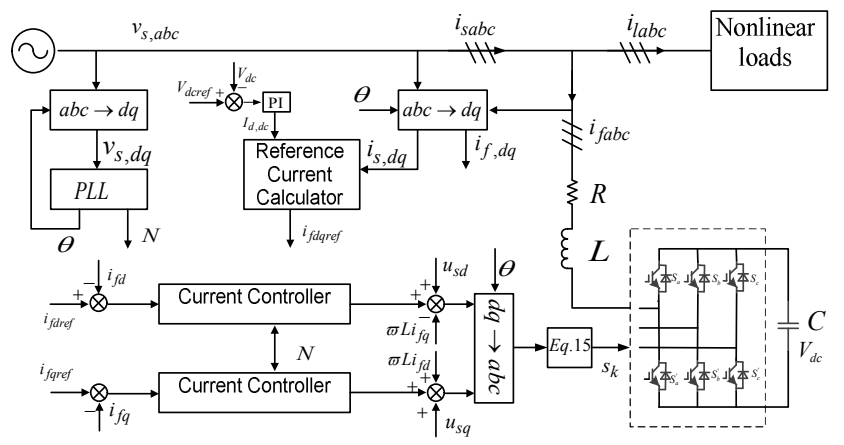

(b)

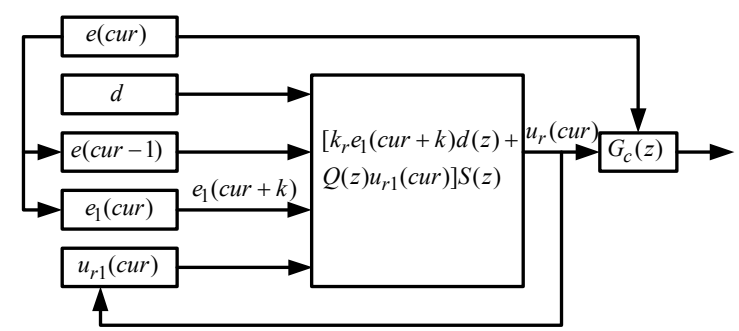

(c)

Fig. 6. Three-phase shunt APF: (a) Circuit structure of shunt APF with LCL filter; (b) Block diagram of the proposed control scheme; (c) The implementation of proposed repetitive controller in DSP.

\subsection{Mathematical model of three-phase shunt APF}

The L and LCL output filters have similar magnitude frequency characteristics within low frequency range [25]. So, the LCL filter behaves as L filter with an inductance value of $L=L_{g f}+L_{f f}$. The general Kirchoff's laws for voltages and currents, as applied to the shunt APF as shown in Fig. 6(a), it provides with differential equation in stationary $a b c$ frame as follow:

$$
\begin{gathered}
L \frac{d i_{f k}}{d t}=u_{s k}-R i_{f k}-u_{f k} \\
u_{f k}=V_{d c} s_{k}
\end{gathered}
$$

where $k=a, b, c$, the switching function $s_{k}$ of the $k$ th leg of the boost converter is defined as:

$$
s_{k}=\left\{\begin{array}{c}
1, \text { if } \mathrm{S}_{\mathrm{k}} \text { is on and } \mathrm{S}_{\mathrm{k}}^{\prime} \text { is off } \\
-1, \text { if } \mathrm{S}_{\mathrm{k}} \text { is off and } \mathrm{S}_{\mathrm{k}}^{\prime} \text { is on }
\end{array}\right.
$$

The transformation matrix from the stationary reference frame to the synchronous reference frame is

$$
T=\sqrt{\frac{2}{3}}\left[\begin{array}{ccc}
\sin \varpi t & \sin \left(\varpi t-\frac{2}{3} \pi\right) & \sin \left(\varpi t+\frac{2}{3} \pi\right) \\
-\cos \varpi t & -\cos \left(\varpi t-\frac{2}{3} \pi\right) & -\cos \left(\varpi t+\frac{2}{3} \pi\right)
\end{array}\right]
$$

Where $\varpi=2 \pi f$ is the fundamental angular frequency. By multiplying matrix $T^{-1}$ on both sides of (14)-(15), in $d q$ frame, they will be

$$
\begin{gathered}
L \frac{d i_{f d}}{d t}=u_{s d}-R i_{f d}+\varpi L i_{f q}-u_{f d} \\
L \frac{d i_{f q}}{d t}=u_{s q}-R i_{f q}-\varpi L i_{f d}-u_{f q} \\
C \frac{d V_{d c}}{d t}=s_{d} i_{f d}+s_{q} i_{f q}
\end{gathered}
$$

There are cross-coupling items exist between the $d-$ and $q-$ axis [26]. The controller, as shown in Fig. 6(b), with feedback cross-decoupling can eliminate the coupling between the $d-$ and $q$-axis. The grid voltage feedforward path is added to eliminate the influence of grid voltage fluctuation.

\subsection{PLL}

In the proposed repetitive control scheme, the time delay coefficient $d$ is updated online relying on the real-time grid frequency, which is measured by PLL [27]. The accuracy of the PLL, as shown in Fig. 6(b), will affect the performance of the control system. Therefore, the output error of the PLL should be as small as possible.

The bandwidth is much slower than the inner current loop and is much faster than the grid frequency. The coefficient $d$ will change only a very little bite and can be treated as constant within a control period, so the proposed repetitive control scheme can be analysis in stability. In practical application, the grid voltage is not pure sinusoidal, which may affect the output accuracy of the PLL. To overcome this problem, a low pass filter (LPF) is introduced to make its output contains only dc component, which is used as the input of the PLL.

The PLL is calculated at the sampling rate, for example $9.6 \mathrm{kHz}$. In this paper, a first-order Pade approximants is chosen, which means that only two coefficients need to be calculated online. It will just take a little time for the 144 MHz DSP. 


\section{Simulation and Experimental Validation}

\subsection{Controller design}

The transfer function of the control plant is

$$
G_{p}(z)=\frac{0.2277 z^{2}+0.1343 z-0.002029}{z^{3}-0.6189 z^{2}-0.3086 z-0.07244}
$$

The PI feedback controller is chosen as

$$
G_{f}(z)=1.25+\frac{0.0021 z}{z-1}
$$

The core of $Q(z)$ is to widen the system stability margin by reducing the gains of repetitive controller at high frequency range. For higher cut-off frequency and wide bandwidth $\alpha_{0}$ should be as large as possible. So the $Q(z)$ is selected as:

$$
Q(z)=\frac{z+8+z^{-1}}{10}
$$

According to theoretical analysis and experimental verification, the repetitive controller gain $k_{r}=0.9$, the phase lead term $z^{k}$ is designed with $k=3$.

The output of the PLL and the coefficients of the proposed FIR filter are both calculated at $9.6 \mathrm{kHz}$. The PI regulator of the PLL can be designed as

$$
\frac{0.2338 z-0.2327}{z-1}
$$

For the proposed repetitive control of (11), $m=1$ is chosen to be the Pade approximants order. Hence the corresponding delay time item will be

$$
z^{-N}=z^{-192} \frac{(1-d)+(1+d) z^{-1}}{(1+d)+(1-d) z^{-1}}
$$

where $d \in[-1.901,1.939]$.

\subsection{Simulation results}

A simulation model of the shunt APF system has been built in MATLAB/Simulink to verify the effectiveness of the proposed control scheme. The detailed system parameters are given in Table 1. In order to validate the effectiveness of the proposed repetitive control scheme, the simulation results of conventional repetitive control are presented for comparison.

Fig. 7 shows the grid current without shunt APF and with a proposed scheme-controller shunt APF at $50 \mathrm{~Hz}$. The value of total harmonic distortion (THD) is reduced from
Table 1. System parameters

\begin{tabular}{c|c|c}
\hline Symbol & Parameters & Values \\
\hline$u_{s k}$ & Grid voltage (line-neutral) & $220 \mathrm{~V}$ \\
\hline$f$ & Nominal grid frequency & $50 \mathrm{~Hz}$ \\
\hline$L_{f f}$ & Converter-side inductance & $160 \mu \mathrm{H}$ \\
\hline$L_{g f}$ & Grid-side inductance & $260 \mu \mathrm{H}$ \\
\hline$C_{f}$ & Filter capacitance & $18 \mu \mathrm{F}$ \\
\hline$R_{d}$ & Filter damping resistor & $2.5 \Omega$ \\
\hline$R$ & Equivalent resistance of the inductors & $0.05 \Omega$ \\
\hline$V_{d c}$ & Dc-link voltage & $720 \mathrm{~V}$ \\
\hline$C$ & Dc-link capacitance & $10000 \mu \mathrm{F}$ \\
\hline$f_{s}$ & Nominal switching frequency & $9.6 \mathrm{KHz}$ \\
\hline
\end{tabular}
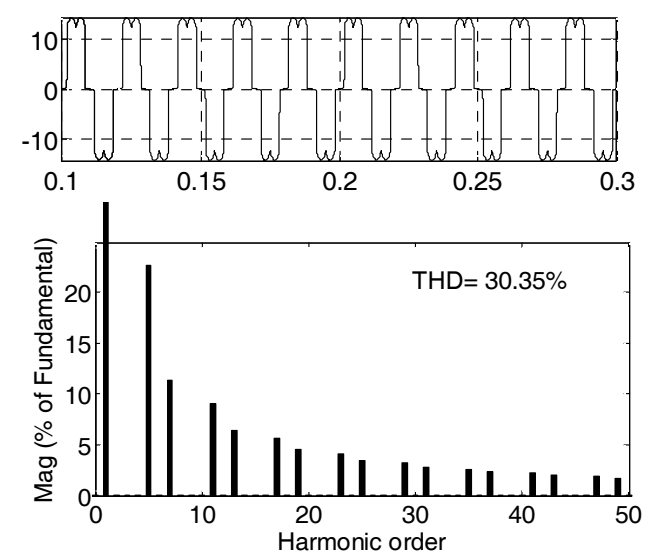

(a)
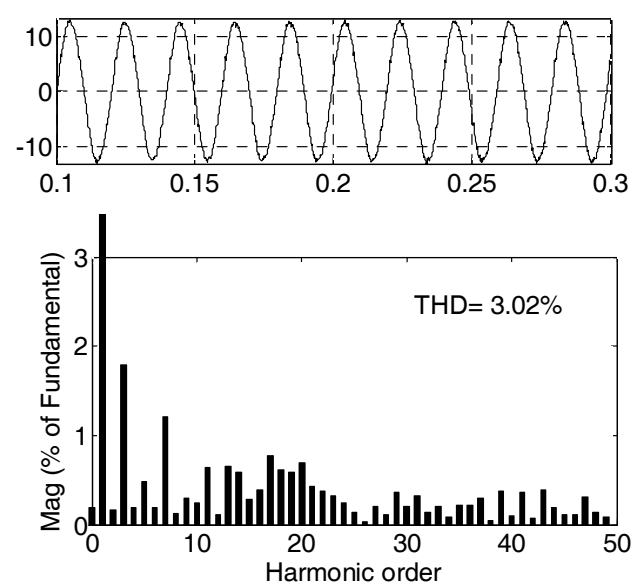

(b)

Fig. 7. Steady-state responses at $50 \mathrm{~Hz}$. Grid current $i_{s a}$. Harmonic spectrum of $i_{s a}$. (a) Without a shunt APF. (b) With a proposed scheme-controlled shunt APF.

$30.35 \%$ to $3.02 \%$. The grid current and its harmonic spectrum at $49.5 \mathrm{~Hz}$ and $50.5 \mathrm{~Hz}$ are shown in Fig. 8 and Fig. 9, respectively. The THD of grid frequency at $49.5 \mathrm{~Hz}$ is $3.16 \%$ with the proposed repetitive control scheme, compared to $6.83 \%$ with the traditional one. At $50.5 \mathrm{~Hz}$, they are $3.14 \%$ and $6.96 \%$, respectively. From the simulated results, the conventional repetitive controller heavily relies on the value of grid frequency, while the improved repetitive controller always keeps a good 

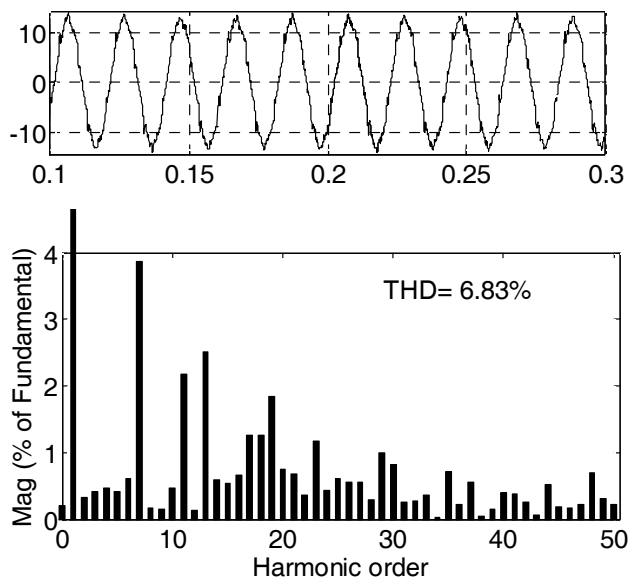

(a)
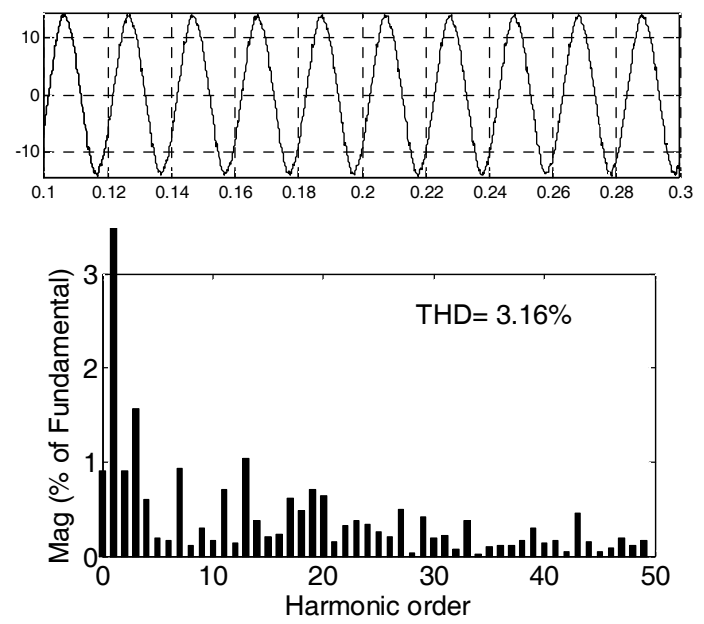

(b)

Fig. 8. Steady-state responses at $49.5 \mathrm{~Hz}$. Grid current $i_{s a}$. Harmonic spectrum of $i_{s a}$. (a) With conventional repetitive control scheme. (b) With proposed repetitive control scheme.

performance at different grid frequency. So, the proposed repetitive controller provides better steady-state performance than the conventional one.

\subsection{Experimental results}

A shunt APF experimental system has been built in our laboratory, as shown in Fig. 10, with the system parameters are given in Table 1. The control algorithm was implemented based on a 32-bit floating-point DSP (TMS320C6747 by Texas Instruments). The grid voltage is generated by a programmable ac power source. The experimental analysis instruments are Oscilloscope and power quality analyzer.

Fig. 11 shows the grid voltage, grid current and harmonic spectrum of $i_{s a}$ at $50 \mathrm{~Hz}$ without shunt APF. Fig. 12 shows the grid voltage, grid current and harmonic spectrum of $i_{s a}$ at $50 \mathrm{~Hz}$ with PI current controller. Figs. 11-12 indicate that the value of THD be reduced from $28.42 \%$ to
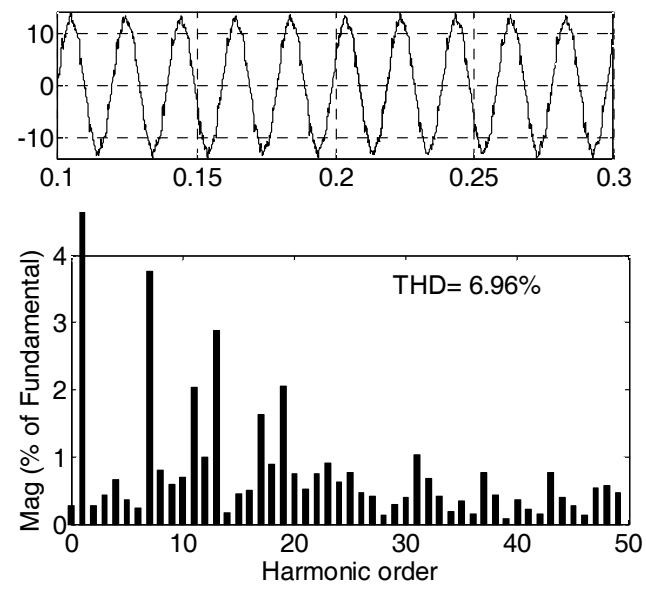

(a)
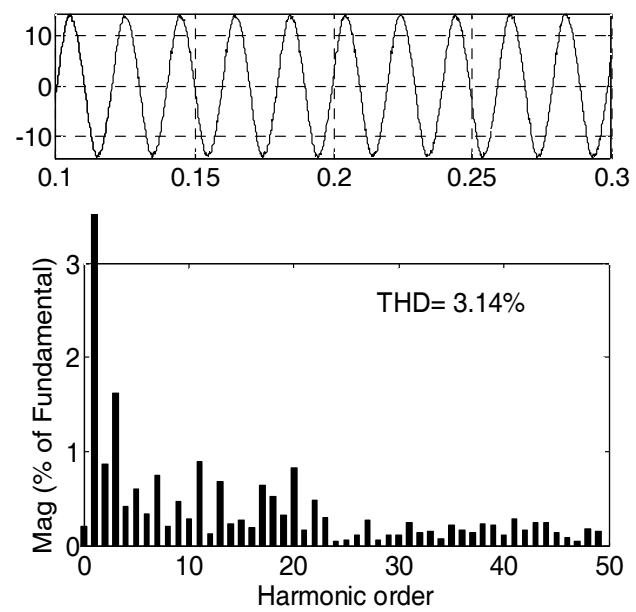

(b)

Fig. 9. Steady-state responses at $50.5 \mathrm{~Hz}$. Grid current $i_{s a}$. Harmonic spectrum of $i_{s a}$ : (a) With conventional repetitive control scheme; (b) With proposed repetitive control scheme.

$14.46 \%$ with the help of PI current controller alone. As mentioned earlier, the PI current controller is unable to effectively compensate the harmonic currents due to the bandwidth limitation and delay time. Fig. 13 shows the steady-state response with proposed scheme-controlled shunt APF at $50 \mathrm{~Hz}$ : the grid voltage, the grid current, and the harmonic spectrum of $i_{s a}$, where the number of sampling point $N=192$. Figs. $11-13$ imply that the total harmonic distortion (THD) of grid current from $28.42 \%$ to $3.17 \%$ with proposed repetitive controller. The control performance hasn't improved compare to the conventional one.

Fig. 14 shows the grid current $i_{s a}$ and its harmonic spectrum at $49.5 \mathrm{~Hz}$ with conventional repetitive control scheme and proposed repetitive control scheme, respectively. The number of sampling point $N=193.9$, under the grid frequency is $49.5 \mathrm{~Hz}$. Fig. 14 implies that the THD of grid current from $28.42 \%$ to $6.71 \%$ with conventional repetitive 


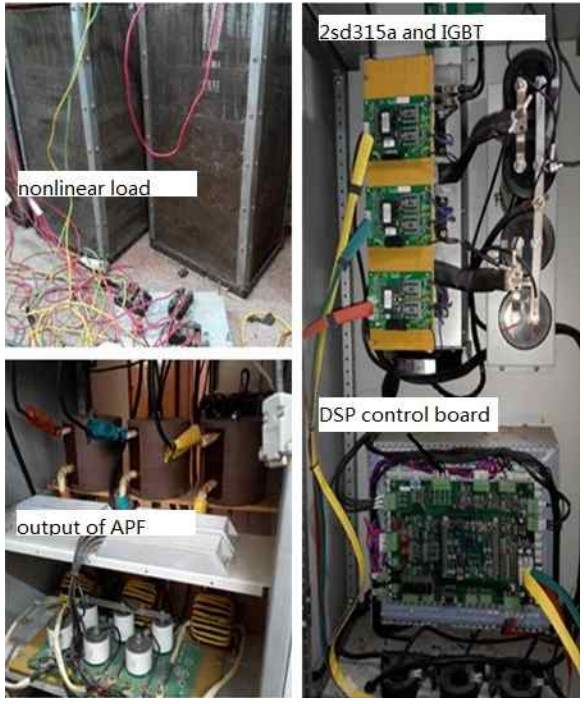

Fig. 10. Experimental system of three-phase shunt APF.

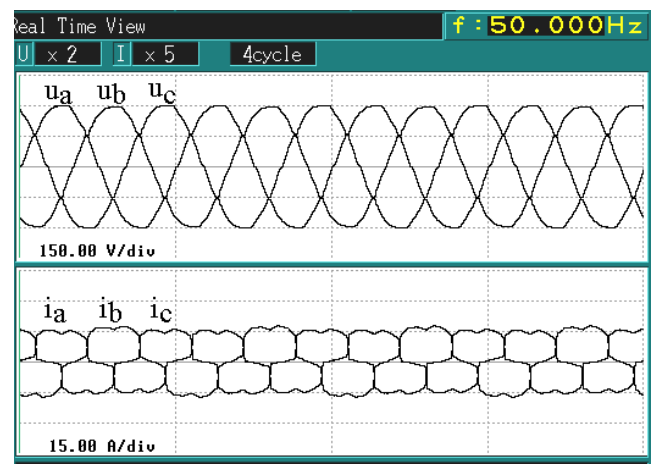

(a)

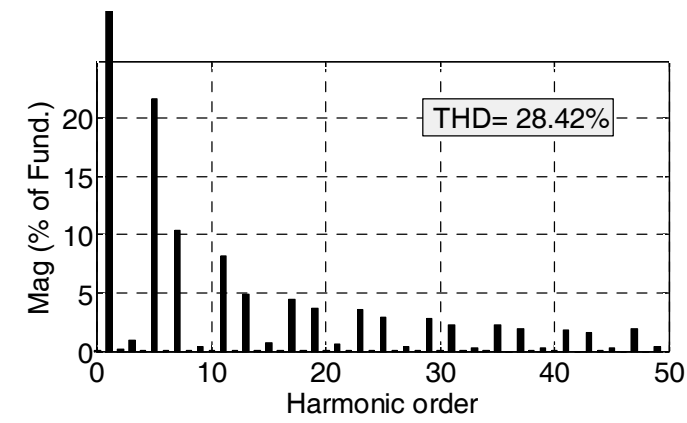

(b)

Fig. 11. Steady-state performance at $50 \mathrm{~Hz}$ without a shunt APF: (a) Grid voltage $u_{s a}$ and grid current $i_{s a}$; (b) Harmonic spectrum of $i_{s a}$.

control and to $3.35 \%$ with the proposed repetitive control scheme.

Fig. 15 shows the grid current $i_{s a}$ and its harmonic spectrum at $50.5 \mathrm{~Hz}$ with conventional repetitive control scheme and proposed repetitive control scheme, respectively. The number of sampling point $N=190.1$, under the grid frequency is $50.5 \mathrm{~Hz}$. Fig. 15 implies that the THD of grid

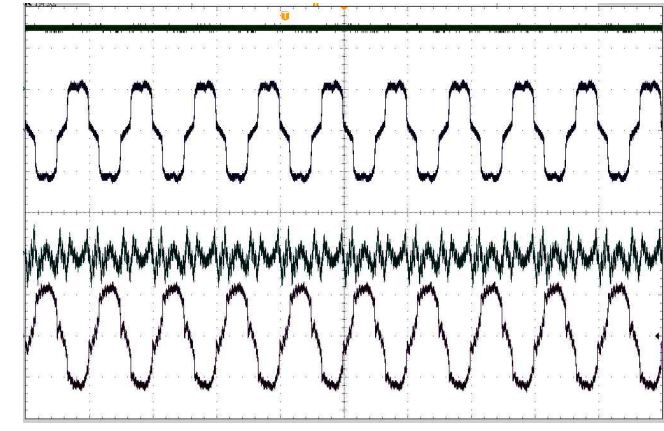

(a)

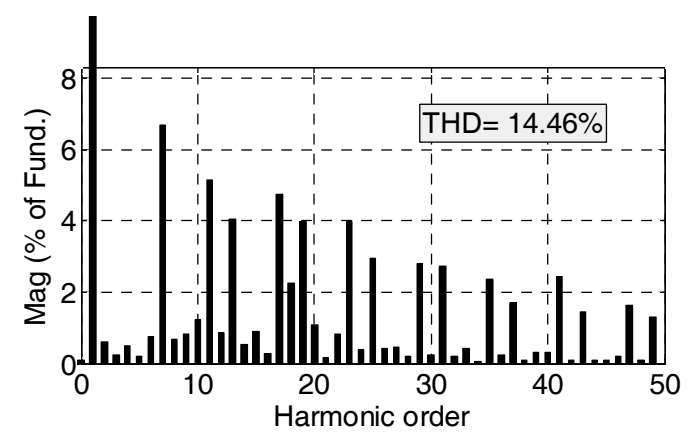

(b)

Fig. 12. (a) Steady-state performance at $50 \mathrm{~Hz}$ with PI control. (b) Harmonic spectrum of $i_{s a}$.

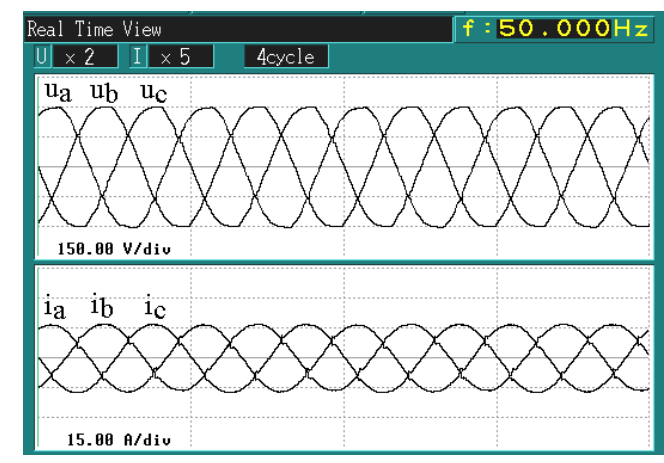

(a)

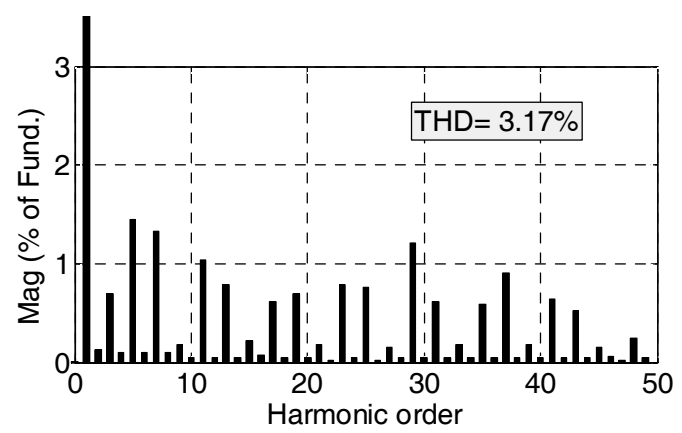

(b)

Fig. 13. Steady-state performance at $50 \mathrm{~Hz}$ with a proposed-controlled shunt APF: (a) Grid voltage and grid current; (b) Harmonic spectrum of $i_{s a}$. 


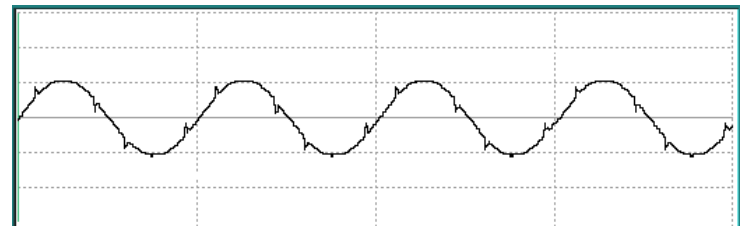

(a)

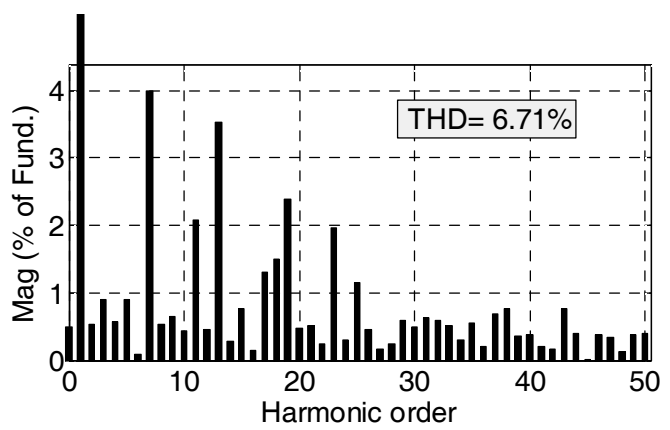

(b)

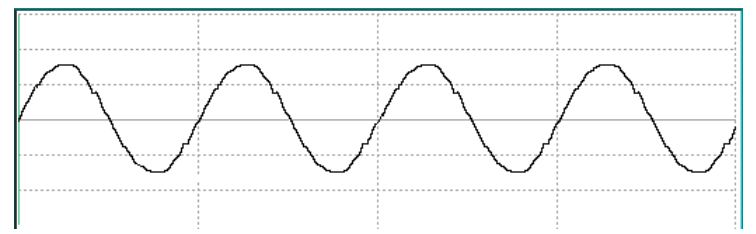

(c)

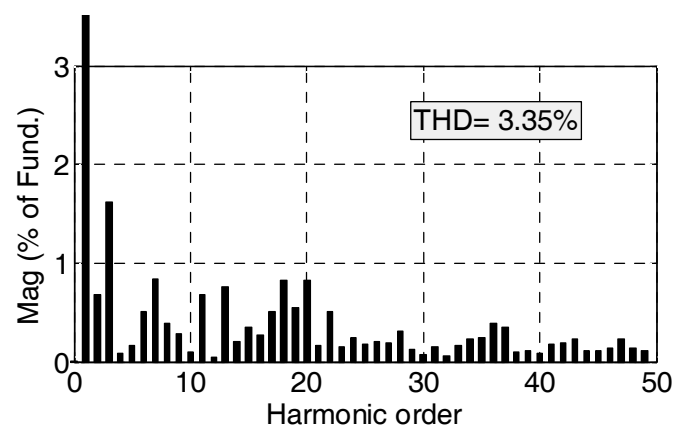

(d)

Fig. 14. Steady-state performance at $49.5 \mathrm{~Hz}$. (a) Grid current $i_{s a}$ with conventional repetitive control scheme. (b) Harmonic spectrum of $i_{s a}$ with conventional repetitive. (c) Grid current $i_{s a}$ with proposed repetitive control scheme. (d) Harmonic spectrum of $i_{s a}$ with proposed repetitive control.

current from $28.42 \%$ to $7.04 \%$ with conventional repetitive control and to $3.29 \%$ with the proposed repetitive control scheme.

Fig.16 shows the THD value of grid current $i_{s a}$ under grid frequency from $49.5 \mathrm{~Hz}$ to $50.5 \mathrm{~Hz}$ with conventional repetitive control scheme and proposed repetitive control scheme, respectively. The experimental results, as shown in Figs. 13-16, demonstrate that the proposed repetitive control scheme have better steady-state performance under variable grid frequency.

Fig. 17 shows the dynamic performance with the

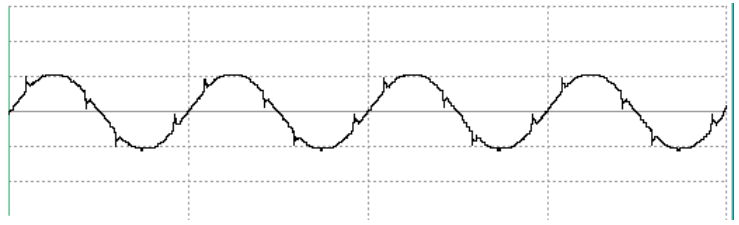

(a)

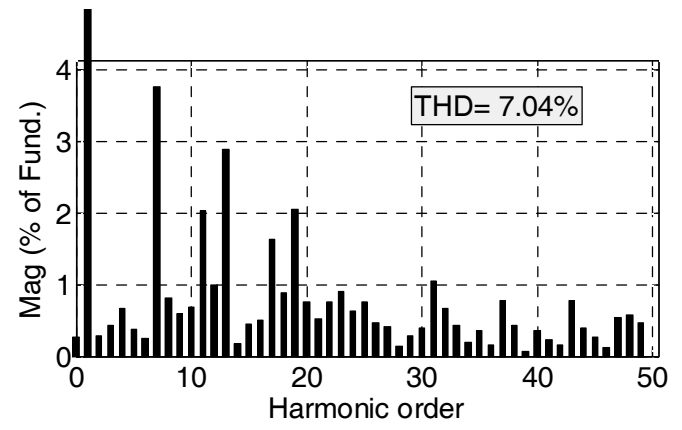

(b)

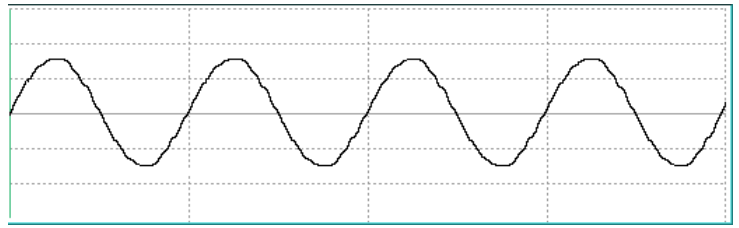

(c)

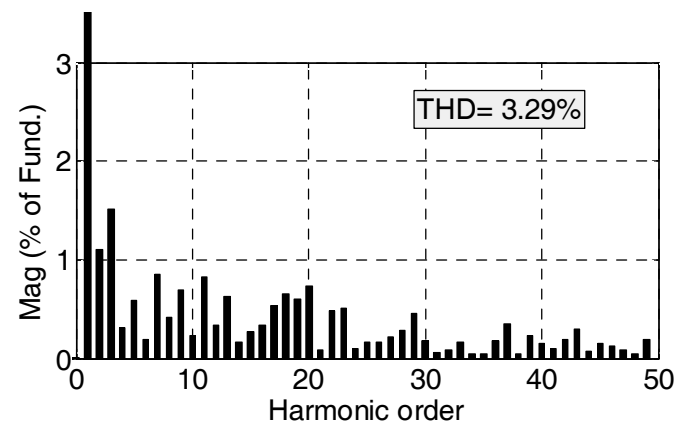

(d)

Fig. 15. Steady-state performance at $50.5 \mathrm{~Hz}$. (a) Grid current $i_{s a}$ with conventional repetitive control scheme. (b) Harmonic spectrum of $i_{s a}$ with conventional repetitive. (c) Grid current $i_{s a}$ with proposed repetitive control scheme. (d) Harmonic spectrum of $i_{s a}$ with proposed repetitive control.

proposed frequency-adaptive repetitive control while the grid frequency changes from $49.5 \mathrm{~Hz}$ to $50.5 \mathrm{~Hz}$.

\section{Conclusion}

In this paper, an improved repetitive control scheme with frequency adaptive capability for three-phase shunt APF has been presented. The variable-delay items in repetitive control are approximated by Pade approximants. The proposed repetitive control scheme can achieve zero 


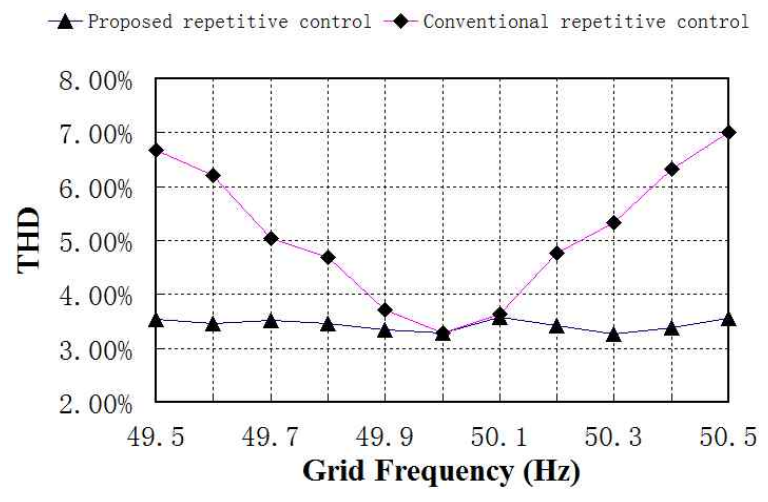

Fig. 16. THD of grid current $i_{s a}$ from $49.5 \mathrm{~Hz}$ to $50.5 \mathrm{~Hz}$.

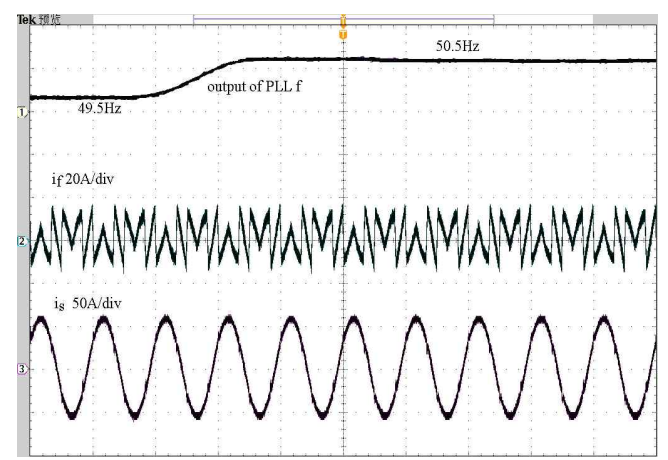

Fig. 17. Dynamic response to change of the grid frequency from $49.5 \mathrm{~Hz}$ to $50.5 \mathrm{~Hz}$.

steady-state error tracking of any periodic signal with variable grid frequency at the fixed sampling rate. The criteria of stability proposed repetitive control system is given, which is similar to the conventional repetitive control system. Simulation and experimental results demonstrated that the proposed repetitive control scheme is an effective solution to improve the tracking performance and reduce the harmonic distortion during grid frequency variation.

\section{References}

[1] H. Akagi, "New trends in active filters for power Cconditioning," IEEE Trans. Ind. Appl., vol. 32, no. 2, pp. 1312-1332, Nov./Dec. 1996.

[2] F. Z. Peng, "Application issues of active power filters," IEEE Ind. Appl. Mag., vol. 4, no. 5, pp. 21-30, Sep./Oct. 1998.

[3] S. Buso, L. Malesani and P. Mattavelli, "Comparison of current control techniques for active filters applications," IEEE Trans. Ind. Electron., vol. 45, no. 5, pp. 722-729, Oct. 1998.

[4] S. Rahmani, N. Mendalek and K. Al-Haddad, "Experimental design of a nonlinear control technique for three-phase shunt active power filter," IEEE Trans. Ind. Electron., vol. 57, no. 10, pp. 3364-3374,
Oct. 2010.

[5] R. I. Bojoi, G. Griva, V. Bostan, M. Gurriero, F. Farina and F.Profumo, "Current control strategy for power conditioners using sinusoidal signal integrators in synchronous reference frame," IEEE Trans. Power Electron., vol. 20, no. 6, pp. 1402-1412, Nov. 2005.

[6] C. Lascu, L. Asiminoaei, I. Boldea and F. Blaabjerg, "Frequency response analysis of current controllers for selective harmonic compensation in active power filters," IEEE Trans. Ind. Electron., vol. 56, no. 2, pp. 337-347, Feb. 2009.

[7] R. Griñó and R. Costa-Castelló, "Digital repetitive plug-in controller for odd-harmonic periodic references and disturbances," Automatica, vol. 41, no. 1, pp. 153-157, Jan. 2005.

[8] R. Griñó and R. Costa-Castelló, "Digital Repetitive Control of a Three-Phase Four-Wire Shunt Active Filter," IEEE Trans. Ind. Electron., vol. 54, no. 4, pp. 1495-1503, Jun. 2007.

[9] B. Francis, W. Wonham, "Internal model principle in control theory," Automatical, 1976.

[10] K. Zhang, Y. Kang, J. Xiong and J. Chen, "Direct repetitive control of SPWM inverters for UPS purpose," IEEE Trans. Power Electron., vol. 18, no.3, pp. 784-792, May 2003.

[11] K. Zhou, D. Wang, and G. Xu, "Repetitive controlled three-phase reversible PWM rectifier," In Proc. Amer. Control Conf., vol. 1, pp. 125-129, 2000.

[12] Keliang Zhou and Danwei Wang, "Digital repetitive controlled three-phase PWM rectifier," IEEE Trans. Ind. Electron., vol. 48, no. 4, pp. 820-830, Aug. 2001.

[13] Quoc-Nam Trinh and Hong-Hee Lee, "Advanced repetitive controller to improve the voltage characteristics of distributed generation with nonlinear loads," Journal of Power Electronics, vol. 13, no. 3, pp. 409-418, May. 2013.

[14] Rabia Nazir, Keliang Zhou, Neville R. Watson, et al., "Frequency adaptive repetitive control of gridconnected inverters," 2014 International Conference on Control, Design and Information Technologies, pp. 584-588, 2014.

[15] Yigang, Danwei Wang, Bin Zhang, Keliang Zhou, "Fractional delay based repetitive control with application to PWM DC/AC converters," $16^{\text {th }}$ IEEE International Conference on Control Applications, pp. 928-933, Singapore, 1-3 Oct. 2007.

[16] T. Hornik and Q. C. Zhang, "H $\infty$ repetitive voltage control of grid-connected inverters with a frequency adaptive mechanism," IET Power Electron., vol. 3, no. 6, pp. 925-935, Nov. 2010.

[17] T. Hornik and Q. C. Zhang, "A current-control strategy for voltage-source inverter in microgrids based on $\mathrm{H} \infty$ and repetitive control," IEEE Trans. Power Electron., vol. 26, no. 3, pp. 943-952, Mar. 2011.

[18] Dong Chen, Junming Zhang, Zhaoming Qian, “An 
improved repetitive control scheme for grid-connected inverter with frequency-adaptive capability," IEEE Trans. Ind. Electron., vol. 60, no. 2, pp. 814823, Feb. 2013.

[19] Dong Chen, Junming Zhang, Zhaoming Qian, "Research on fast transient and $6 n \pm 1$ harmonics suppressing repetitive control scheme for three-phase grid-connected inverters," IET Power Electron., vol. 6, no. 3, pp. 601-610, 2013.

[20] Zixiang Zou, Zheng Wang, "Fractional-order repetitive control of programmable AC power sources," IET Power Electron., vol. 7, no. 2, pp. 431438, 2014.

[21] Ali Asbafkan, Behzad Mirzaeeian, Mehdi Niroomand, et al., "Frequency adaptive repetitive control of grid connected inverter for wind turbine applications," Electrical Engineering, 21st Iranian Conference on, 2013.

[22] Yongheng Yang, Keliang Zhou, Huai Wang, "Frequency adaptive selective harmonic control for gridconnected inverters," IEEE Trans. Power Electron., vol. 30, no. 7, pp. 3912-3924, July, 2011.

[23] P. Tomei, C. M. Verrelli, "Linear repetitive learning controls for nonlinear systems by Pade approximants," Int. J. Adapt. Control Signal Processing, vol. 29, pp. 783-804, 2015.

[24] G. F. Franklin, J. D. Powell, A. Emami Naeini, "Feedback Control of Dynamic Systems," Reading, MA: Addison-Wesley, 1991.

[25] Zheng Zeng, Jia-Qiang Yang, Shi-Lan Chen, "Fsattransient repetitive control strategy for a three-phase LCL filter-based shunt active power filter," Journal of Power Electronics, vol. 14, no. 2, pp. 392-401, Mar. 2014.

[26] Ye Y, Kazerani M, and Quintana V H, "A novel modeling and control method for three-phase PWM converters," PESC. 2001, IEEE 32th Annual. 2001, 1: 102-107.

[27] S. K. Chung, "A phase tracking system for three phase utility interface inverters," IEEE Trans. Power Electron., vol. 15, no. 3, pp. 431-438, May 2000.

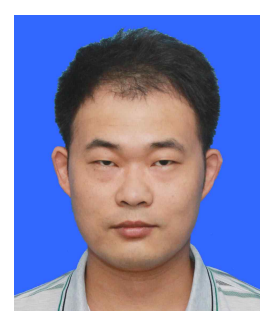

Biaoguang Sun was born in Xinxiang, Henan Province, China, in 1984. He received his B.S. and M.S. degrees in Automation from Henan Polytechnic University, Jiaozuo, China in 2008 and 2011, respectively. He is currently working toward a Ph.D. in Electrical Engineering at the South China University of Technology, Guangzhou, China. His research interests include high-power density rectifiers, active power filter, and distributed power quality.

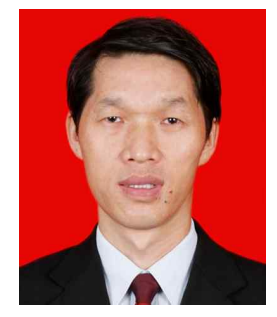

Yunxiang Xie was born in Shaoyang, Hunan Province, China in 1965. He received his B.S., M.S., and Ph.D. degrees in Electrical Engineering from Xian Jiaotong University, Xian, China in 1985, 1988, and 1991, respectively. He was a postdoctoral researcher from 1991 to 1994 and an associate professor from 1994 to 1999 in the School of Electrical Power, South China University of Tech, Guangzhou, China. He has been a professor of the same department at the South China University of Tech since 1999. He is a senior member of the China Electro technique Society and the director of the Electrical Energy-saving Special Committee of the China Electro technical Society. While teaching in the Chinese Society of Mechanical Education and Chinese Power Industry Education Association of Electrical Engineering and Automation Committee, he authored or coauthored more than 170 published technical papers. His research areas include power electronics and control, including high-power density rectifiers, multilevel converters, active filter, and distributed power quality and control strategies.

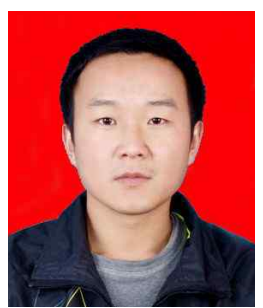

Hui Ma was born in Kaifeng, Henan Province, China in 1985. He received the M.S. degree in Electrical Engineering and Automation from Chang Chun University of Technology, Jilin, China in 2013. He is currently working toward his Ph.D. in Electrical Engineering at the South China University of Technology, Guangzhou, China. His research interests include high-power density rectifiers, multilevel converters, and control strategies.

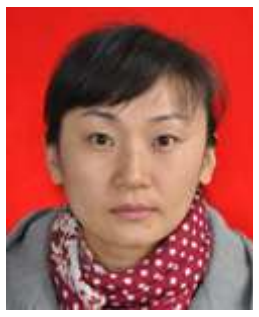

Li Cheng receives the B.S degree in Electrical Automation Technology from Jinan University in 2001 and M.S degree in control theory and engineering from Guangxi University in 2004, then worked in Guizhou University from 2004. She is working toward her Ph.D degree in power electronics from the South China University of Technology. Her interests are new and renewable energy system designs and developments of wind power, photovoltaic energy conversion systems. 